\title{
Exome sequencing identifies a de novo mutation in HDAC8 associated with Cornelia de Lange syndrome
}

Lei Feng, Daizhan Zhou, Zhou Zhang, Yun Liu and Yabo Yang

Journal of Human Genetics (2015) 60, 165; doi:10.1038/jhg.2014.113

Correction to: Journal of Human Genetics 2014; 59, 536-539; doi:10.1038/jhg.2014.60; published online 7 August 2014

Since the publication of this article, the authors of the above paper have noticed an error in the description of mutation. The de novo mutation 'c.586A > T; p.M196K' should have been 'c.587A > T; p.M196K'.

The authors apologize for any inconvenience caused. 